\title{
Information Preservation Method For The Case Of Temperature Variation
}

\author{
Shen, C. , Jiang, J. Z. , Fan, J.* \\ Laboratory of High Temperature Gas Dynamics \\ Institute of Mechanics, Chinese Academy of Sciences
}

\begin{abstract}
The information preservation (IP) method is extended in this paper to include the case of temperature variation. The IP velocity $\vec{u}$ and IP energy e (or IP temperature T) are introduced. They migrate with the simulated molecules and are governed by the momentum and energy conservation equations. The method is illustrated on the example of rarefied gas between parallel plates with different temperatures. Comparison of the IP simulation result is made with experimental data, DSMC calculation and solution of non-linear Boltzmann equation.
\end{abstract}

\section{INTRODUCTION}

The unavoidable statistical scatter that is present in most DSMC ${ }^{[1]}$ calculations is generally regarded as the most serious practical and ideological difficulty with the method, and becomes insurmountable for low Mach number flows characterized by a small ratio of macroscopic to molecular velocity. For low-speed flow, useful small macroscopic information may be completely lost in the back ground noises. And as the deviation of fluctuation decreases as the reciprocal of the square root of the sample size, the signal may remain submerged even for rather larger sample sizes which require enormous computation time. In order to solve this problem and make use of the collectivity of simulated molecules in the DSMC method, the information preservation (IP) ${ }^{[2]}$ method was proposed. The essence of the technique is to store besides the ordinary thermal velocity of the simulated molecules, also the so called information velocity, that is to record the collective velocity of the enormous number of real molecules that the simulated molecule represents. Macroscopic velocity and shear stress are obtained from the time or ensemble average of the information velocities of simulated molecules in a cell or on a wall element. The method has been applied to unidirectional flows such as Couette flow, Poiseuille flow and Rayleigh flow. ${ }^{[2]}$ Excellent agreement is obtained with exact solutions of continuum and free molecular flows and the numerical solutions of the linearized Boltzmann equation in transition regime. The technique is being developed to treat the two dimensional and three dimensional low speed rarefied gas flows, ${ }^{[3,4]}$ i.e. the two dimensional flow around a NACA 0012 air foil and the three dimensional flow in a micro-machined membrane particle filter. It is natural to extend

\footnotetext{
* present address: Dept of Aerospace Engineering Univ. of Michigan, Ann Arbor, MI 48109.
} 
the IP technique, enabling it to deal with situation in which temperature varies in the flow field not due to retardment of the flow but due to the transfer of heat from the non-isothermal boundaries. Unfortunately this extension is not a trivial one. The nontriviality is caused by the fact that the average energy flux of a monatomic molecules cross a surface element in a stationary gas is $2 k T T_{n}$ (where $\Gamma_{n}$ is the particle number flux $\Gamma_{n}=n c / 4$, here $\mathrm{n}$ is the number density of the flow and the $\bar{c}$ is the mean molecular speed), whereas the average energy of a monatomic molecule is $3 k T / 2$, so that a simulated molecule carrying an energy of amount $3 k T / 2$ could not reexhibit the physically real energy flux $2 k T T_{n}$. Despite this difficulty in this paper an attempt is made to enable the IP method to include the case of temperature variation, which takes place in some flow situation of micro-electro-mechanicalsystems (MEMS). The present version is known to have a certain error owning to the above mentioned relationship concerning to the energy amount and energy flux. The procedure is outlined in the next section followed by the illustration on the example of temperature and density distribution of rarefied gas between parallel plates with different temperatures. The results are given for rather large temperature difference $\left(\Delta T / T_{0}\right)$, they are in agreement for both heat transfer and density and temperature distribution for large Kn numbers. As for small Kn numbers IP procedure yields density and temperature results in fairly good agreement with DSMC simulation but gives heat transfer results with apparent discrepancy from the DSMC simulation results for small Kn numbers.

\section{OUTLINE OF THE PROCEDURE OF IP METHOD FOR THE CASE OF TEMPERATURE VARIATION}

Any IP procedure is implemented on the basis of, and embedded in a successfully operated DSMC code. Except the usual microscopic (thermal) velocity, macroscopic velocity, density, and temperature introduced in the DSMC method, the IP (information preservation) velocity $\vec{u}$ and IP energy e of the simulated molecule are introduced as well as IP velocity $\vec{U}$, IP density $\rho$ and IP temperature T of a cell which represent the macroscopic properties of a cell. The IP velocity $\vec{u}$ and IP energy e migrate with the simulated molecules and are governed by the momentum and energy conservation equations:

$$
\begin{aligned}
& \iiint \rho \frac{\overrightarrow{D u}}{D t} d V=-\iint \bar{\sigma} \cdot \dot{l} d S \\
& \iiint \rho \frac{D}{D t}\left(\frac{U^{2}}{2}+e\right) d V=-\iint \vec{q} \cdot \vec{l} d S+\iint(\bar{\sigma} \cdot \vec{U}) \cdot \vec{l} d S
\end{aligned}
$$

where conventional notation is used and $\vec{l}$ is the unit normal to the surface. Meanwhile the IP velocity and temperature of a cell are obtained as the weighted averages of the IP values of simulated molecules in the cell. The macroscopic density of a cell is governed by the mass conservation equation:

$$
\iiint(\partial \rho / \partial t) d V=-\iint \rho \vec{U} \cdot \vec{l} d S
$$

For isothermal flows equation (2) can be omitted, and in some cases $\overline{\bar{\sigma}}$ can be simplified with normal pressure alone retained. For slow non-isothermal flows equation (2) is needed and the Burnett terms together with viscous terms in $\overline{\bar{\sigma}}$ need to be incorporated. The concrete procedure and formulation will be specified for each example problem. 


\section{RAREFIED GAS BETWEEN PARALLEL PLATES WITH DIFFERENT TEMPERATURES}

Rarefied gas is confined between two parallel plates at rest (one aligned on $y=-\mathrm{D} / 2$, the other on $\mathrm{y}=\mathrm{D} / 2$ ) with different temperatures $T_{0}-\Delta T$ and $T_{0}+\Delta T$. The Knudsen number $\mathrm{Kn}$ is defined as $\lambda_{0} / D$, where $\lambda_{0}$ is the mean free path at $\mathrm{y}=0$. The study of heat transfer, density and temperature distribution in the gas for a variety of Knudsen numbers have been pursued by many authors. The non-isothermal IP procedure is illustrated on this problem. For simplicity the monatomic molecules are considered. The movement of the simulated molecules, the encounter of them with body surface and the collision between them are simulated by the DSMC method. After a time step $\Delta t$ the simulated molecules move to a new position, and the macroscopic velocity and temperature of a cell are obtained as the weighted averages of the IP velocity $u_{i, k}$ and IP energy $e_{k}^{i}$ of simulated molecules:

$$
\begin{aligned}
U_{i} & =\sum_{k=1}^{N_{s m}} m_{k} u_{i, k} / \sum_{k=1}^{N_{s m}} m_{k} \\
T^{i} & =e^{i} /(0.5 k)=\left(\sum_{k=1}^{N_{s m}} e_{k}^{i} / N_{s m}\right) /(0.5 k) .
\end{aligned}
$$

Here $N_{s m}$ is the total number of the simulated molecules in the cell, $T^{i}$ and $e^{i}$ are the translational thermal temperature and the translational kinetic energy based on the ith component of thermal velocity. The increment of the IP density of a cell during $\Delta t$ is (according to the mass conservation equation (3)):

$$
\Delta n=-(\Delta t / \Delta V) \iint n \vec{U} \cdot \vec{l} d S .
$$

The macroscopic density is updated accordingly. Meanwhile the increments of IP values $\vec{u}$ and $\mathrm{e}^{\mathrm{i}}$ during time step $\Delta t$ are (according to the momentum and energy conservation equations (1), (2)):

$$
\begin{aligned}
& \Delta \vec{u}=-(\Delta / /(m n \Delta V)) \iint p \vec{l} d S \\
& \Delta e^{i}=-(\Delta t /(m n \Delta V)) \iint \vec{q}^{i} \cdot \vec{l} d S, i=1,2,3
\end{aligned}
$$

where $\Delta V$ is the cell volume. The surface integrals are calculated on the upper and lower sides of each cell. The under integral values in (6), (7), (8) are the number flux, normal momentum flux and heat flux, and are calculated from the assumption that the molecules in each cell are in equilibrium having the cell's velocity, density and temperature as their macroscopic characteristics. For example, the heat flux across the boundary of cell $\mathrm{y}=$ const for the kinetic energy of the $\mathrm{y}$-component $(i=2)$ of the thermal velocity is $\left(s=U /(2 k T / m)^{(1 / 2)}\right)$

$$
q_{y}^{i=2}=\frac{m n(2 k T / m)^{(3 / 2)}}{4 \sqrt{\pi}}\left[\left(1+s^{2}\right) \exp \left(-s^{2}\right) \pm \sqrt{\pi} s\left(1.5+s^{2}\right)(1 \pm \operatorname{erf}(s))\right]
$$

whereas that for the energy of the $\mathrm{x}$ and $\mathrm{z}$ components $(i=1, i=3)$ is

$$
q_{y}^{i=1}=q_{y}^{i=3}=\frac{m n(2 k T / m)^{(3 / 2)}}{4 \sqrt{\pi}}\left[\frac{1}{2} \exp \left(-s^{2}\right) \pm \frac{1}{2} \sqrt{\pi} s(1 \pm \operatorname{erf}(s))\right]
$$


In case of $\mathrm{s}=0 \quad q_{y}^{l=2}=k T \Gamma_{n}, q_{y}^{l=1}=q_{y}^{l=s}=0.5 k T \Gamma_{n}$, where $\Gamma_{n}$ is the number flux in a stationary gas: $\Gamma_{n}=n(k T /(2 \pi m))^{(1 / 2)}$. The difference between the expressions for $q_{y}^{t=2}$ and $q_{y}^{l=1}\left(=q_{y}^{i=3}\right)$ is the reason why the kinetic energies for different components of thermal velocity are introduced separately. When taking the surface integral on each side of the cell, the energy flux $q^{i}$ consists of the outlet energy flux from the cell and the inlet energy flux from the neighboring cell, and the pressure $\mathrm{p}$ is obtained as the average of the pressure of the cell and the pressure of neighboring cell. The increments $\Delta \vec{u}$ and $\Delta e^{i}$ are added to the IP values of the simulated molecules in the cell, the updated values are used for the next calculation.

During collision common post-collision IP velocities are calculated as the mass weighted averages of the pre-collision values and are assigned to each of the encountering molecules, and equipartition of energy is applied to the distribution of the post collision IP energies. When reflecting from walls, for specular reflection IP velocity changes its sign of the normal to the wall component only, and for diffuse reflection it gains the wall's velocity; and for $\alpha$ part of all molecules the IP energy gains the values corresponding to the wall temperature and for the rest $(1-\alpha)$ part the IP energy remains unchanged, where $\alpha$ is the accommodation coefficient. The procedure is iterated until convergence is reached. On the surface, the energy exchange is implemented by concrete counting the energy carried by the simulated molecules, so on the boundary the energy flux on the boundary side of the integral in equation (8) is not calculated repeatedly.

As we have seen, in the equilibrium state, the heat flux across a surface with normal of $i=2$ is $\left(\left(0.5 k T^{i=1}+k T^{i=2}+0.5 k T^{i=3}\right) \Gamma_{n}\right.$, so a molecule when going through such a surface carries in average an energy of $0.5 k\left(T^{i=1}+T^{i=3}+2 T^{i=2}\right)$, and the heat exchange of molecule with the wall surface is

$E_{n}=0.5 k\left(\Delta T_{n}^{i=1}+\Delta T_{n}^{i=3}+2 \Delta T_{n}^{i=2}\right)$. During the sampling time $\tau$, the net energy exchange $E$ is the sum of the heat exchange $E_{n}$ of all molecules participating in sampling. The heat transfer to the wall is $q=E /(\tau \cdot \Delta A)$, where $\Delta A$ is the area of the wall surface.

\section{RESULTS AND DISCUSSION}

The problem of rarified gas confined between two parallel plates with different temperatures is simulated by the IP technique described above for a temperature difference of $\Delta T / T_{0}=0.14$ and with accommodation coefficients of the upper and lower plate $\alpha_{+}=\alpha_{-}(=\alpha)=0.826$.

The table I shows the variation of normalized heat flux $Q_{1} / Q_{F M}$ with $\mathrm{Kn}$ in the problem, where $Q_{F M}$ is the analytical result of $Q_{1}$ for $K n=\infty$ given by [5] $\left(Q_{F M}=-\left(2(k / m) T_{0}\right)^{3 / 2} \pi^{-1 / 2} \rho_{0} \alpha(2-\alpha)^{-1} \Delta T / T_{0}\right)^{*}$. The present result is compared with the solution of the Boltzmann equation ${ }^{[5]}$, the result of the moment method ${ }^{[6]}$, and the calculation by DSMC method carried out in this paper. When the Kn number is small, the heat flux of IP method is the smallest among all methods, and the accuracy is not satisfactory. However, when the Kn number is high, the heat flux of IP method is in good agreement with DSMC procedure, and we can anticipate when Knudsen number tends to infinity (i.e. the flow can be regarded as collisionless flow), the results of both methods will tend to the analytical result $\mathrm{Q}_{\mathrm{FM}}$. 
Table I. The normalized heat flow $Q_{1} / Q_{F M}$ for various Knudsen numbers. $\left(\Delta T / T_{0}=0.14\right)$

\begin{tabular}{|l|l|l|l|l|}
\hline $\mathrm{Kn}$ & $\begin{array}{l}\text { IP method } \\
\text { (present paper) }\end{array}$ & $\begin{array}{l}\text { DSMC method } \\
\text { (present paper) }\end{array}$ & $\begin{array}{l}\text { Solution of Boltzmann } \\
\text { equation }^{[5]}\end{array}$ & Moment method \\
\hline 0.0658 & 0.2132 & 0.3018 & 0.2538 & 0.2716 \\
\hline 0.1942 & 0.4230 & 0.5336 & 0.4843 & 0.5239 \\
\hline 0.7582 & 0.7072 & 0.7813 & 0.7558 & 0.8112 \\
\hline 10. & 0.9584 & 0.9684 & & \\
\hline 100. & 0.9865 & 0.9906 & & \\
\hline
\end{tabular}

The density distribution in the gas for different $\mathrm{Kn}$ obtained by the IP procedure is shown in Fig.1 together with the experimental data ${ }^{[7]}$, the solution of the non-linear Boltzmann equation ${ }^{[5]}$ and the result of DSMC simulation obtained in this paper. Density and $Y$ are normalized by the centerline value and $\mathrm{D}$, respectively. In general, the calculation of IP method is in fair agreement with the results of other methods. At about $\mathrm{Kn}=0.5$, the departure of IP result from DSMC calculation and the solution of the non-linear Boltzmann equation is the largest.

Figure 2. shows the temperature distribution of IP method with the solution of the non-linear Boltzmann equation and the result of DSMC simulation (the temperature is normalized by $T_{0}$ ). The agreement among these methods is fair.

Figure 3. shows the density and temperature distribution of IP technique and DSMC method for $\mathrm{Kn}=0.7582$ with $\alpha_{+}=\alpha_{-}(=\alpha)=0.826$ and $\Delta T / T_{0}=0.03$. When the temperature differences $\left(\Delta T / T_{0}\right)$ decrease, the agreement of density and temperature distribution between IP result and DSMC calculation is better.

The above comparison shows that the present IP procedure gives satisfactory results for both heat transfer and density and temperature distribution for $\mathrm{Kn}$ numbers near the free molecule limit. As for small Kn numbers, it can yield density and temperature distribution results in fairly good agreement with DSMC simulations, but can not provide satisfactory heat transfer results.

Of course, the IP procedure has the advantage of prompt convergence which is not so important for the present case, but is crucial for complicated MEMS flows. In this case, when the IP sample size is about 4,000,000 per cell, the convergent result (when the y-component $(i=2)$ of the macroscopic velocity is less than 0.002) is achieved; meanwhile DSMC method should have the sample size of about $1,000,000,000$ per cell to reach convergence.

Unlike the isothermal flow cases where the IP simulation results yield good agreement with DSMC calculation and other exact solutions, the present IP technique for non-isothermal flow provides results only in fairly satisfactory agreement with DSMC simulation and other solutions and for the heat flux of small $\mathrm{Kn}$ number, the agreement is not satisfactory. The technique deserves further development. 


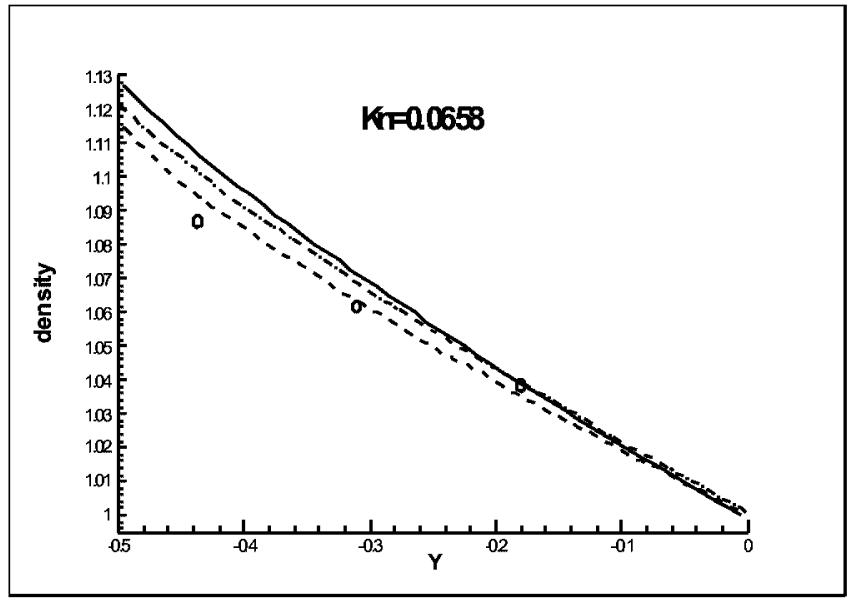

(a)

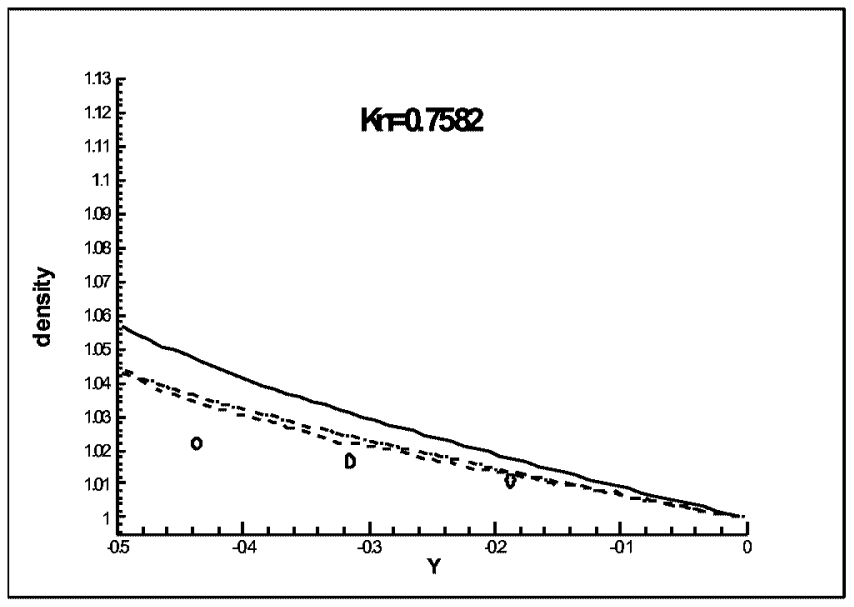

(c)

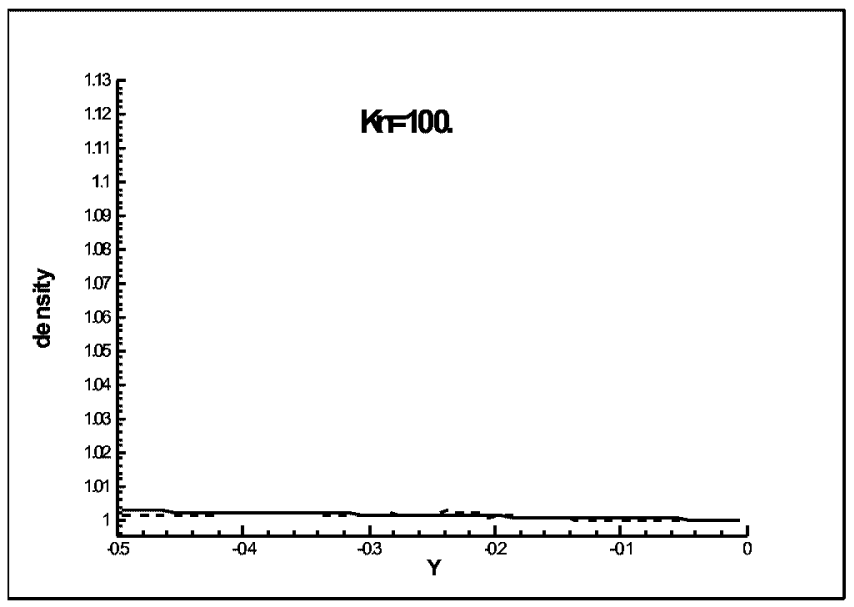

(e)

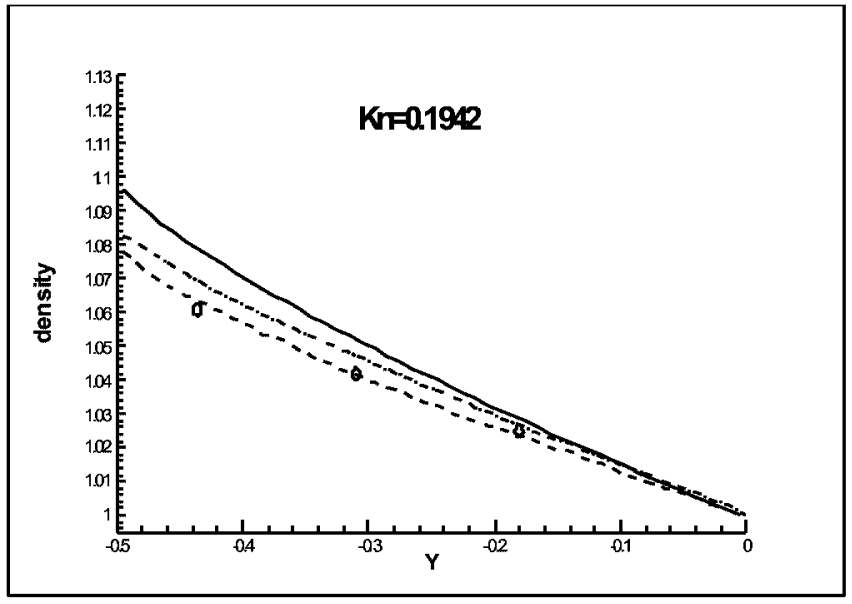

(b)

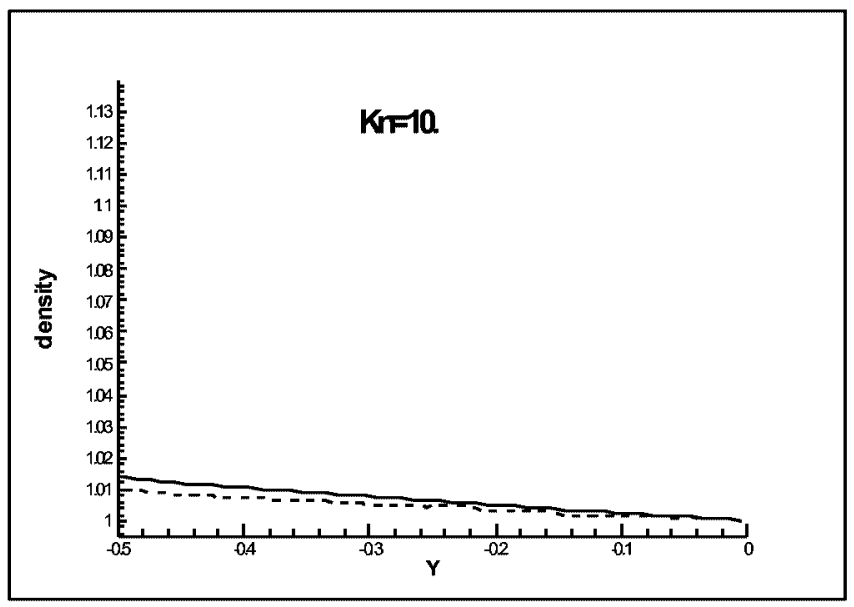

(d)

FIG. 1. Density distribution for $\mathrm{a} \pm=0.826$ and $\Delta T / T_{0}=0.014$. Here, :IP result; $\quad-$ : DSMC calculation; : solution of non-linear Boltzmann equation ${ }^{[5]}$. The symbols ${ }^{\circ}$ indicate the experimental data ${ }^{[7]}$. 


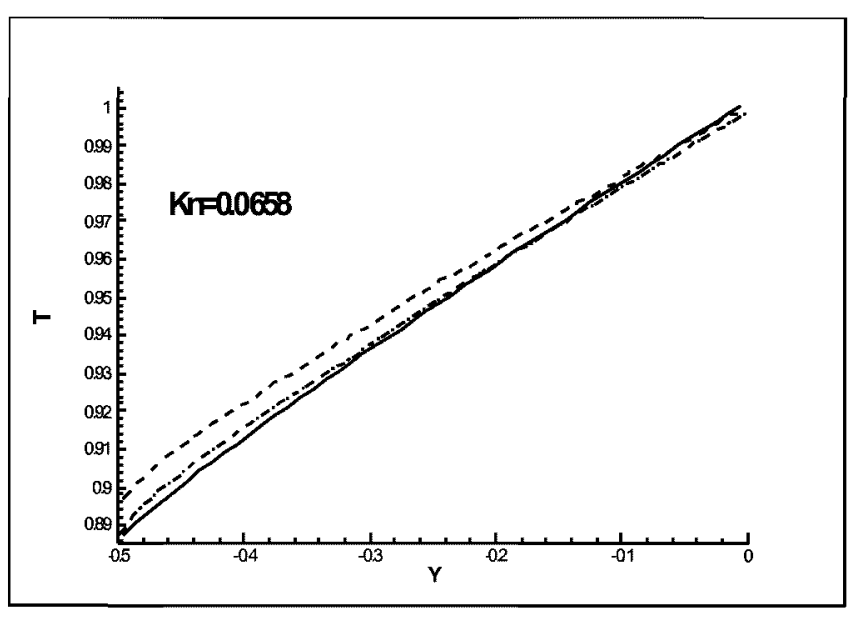

(a)

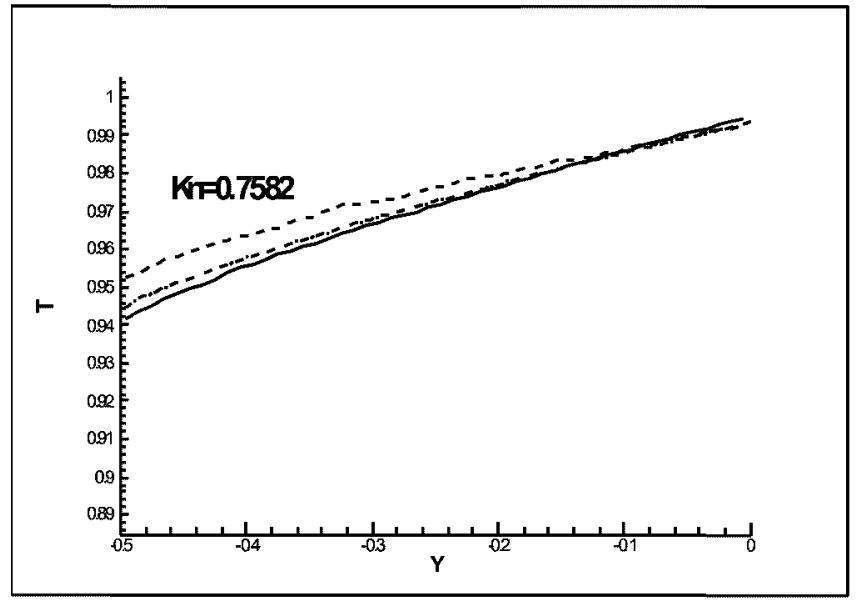

(d)

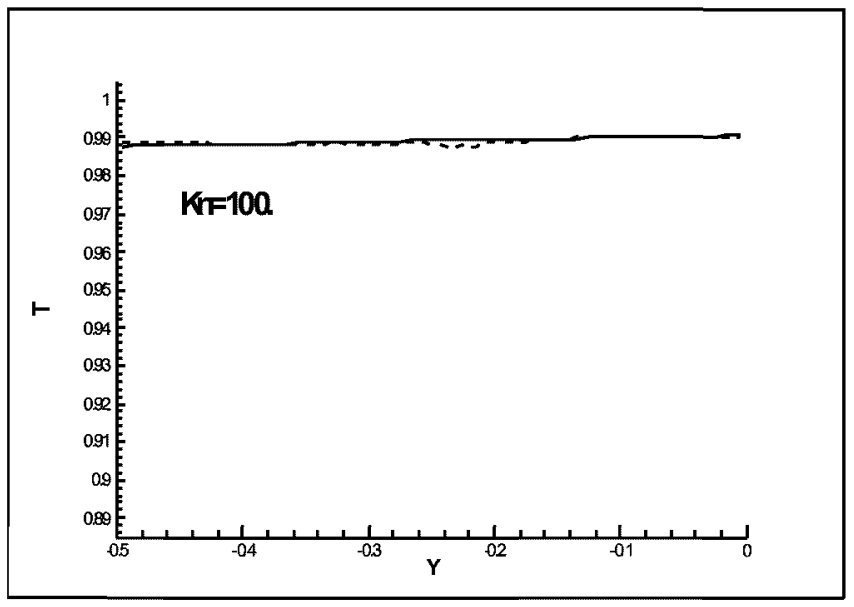

(e)

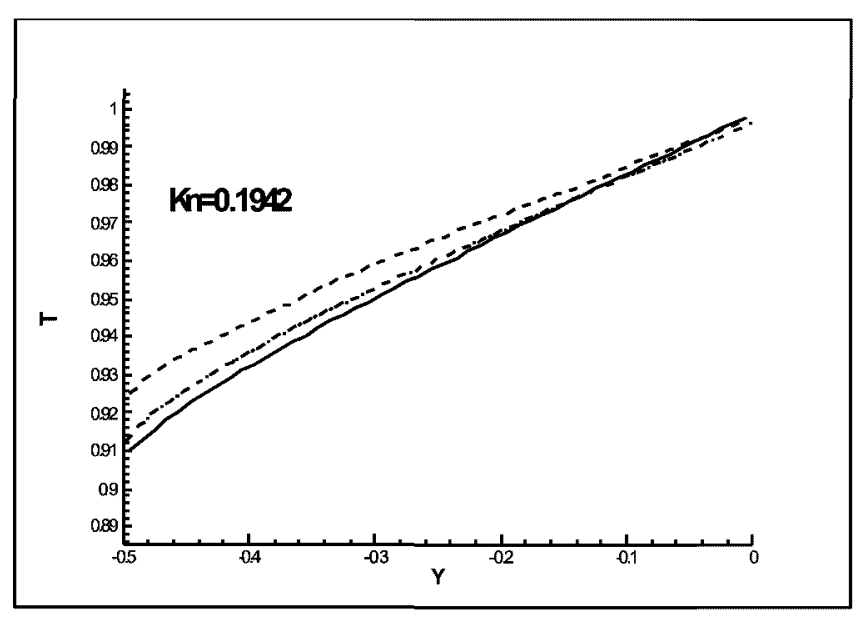

b

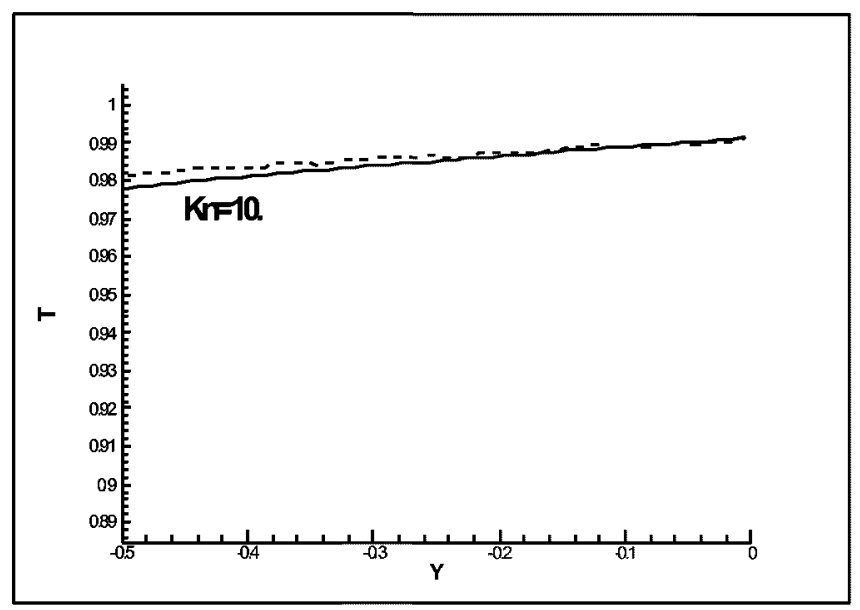

(d)

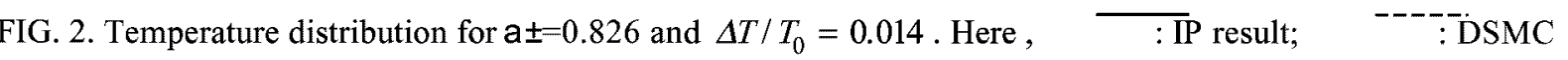
calculation; $-\cdots-$ : solution of non-linear Boltzmann equation ${ }^{[5]}$. The symbols ${ }^{0}$ indicate the experimental data ${ }^{[7]}$. 

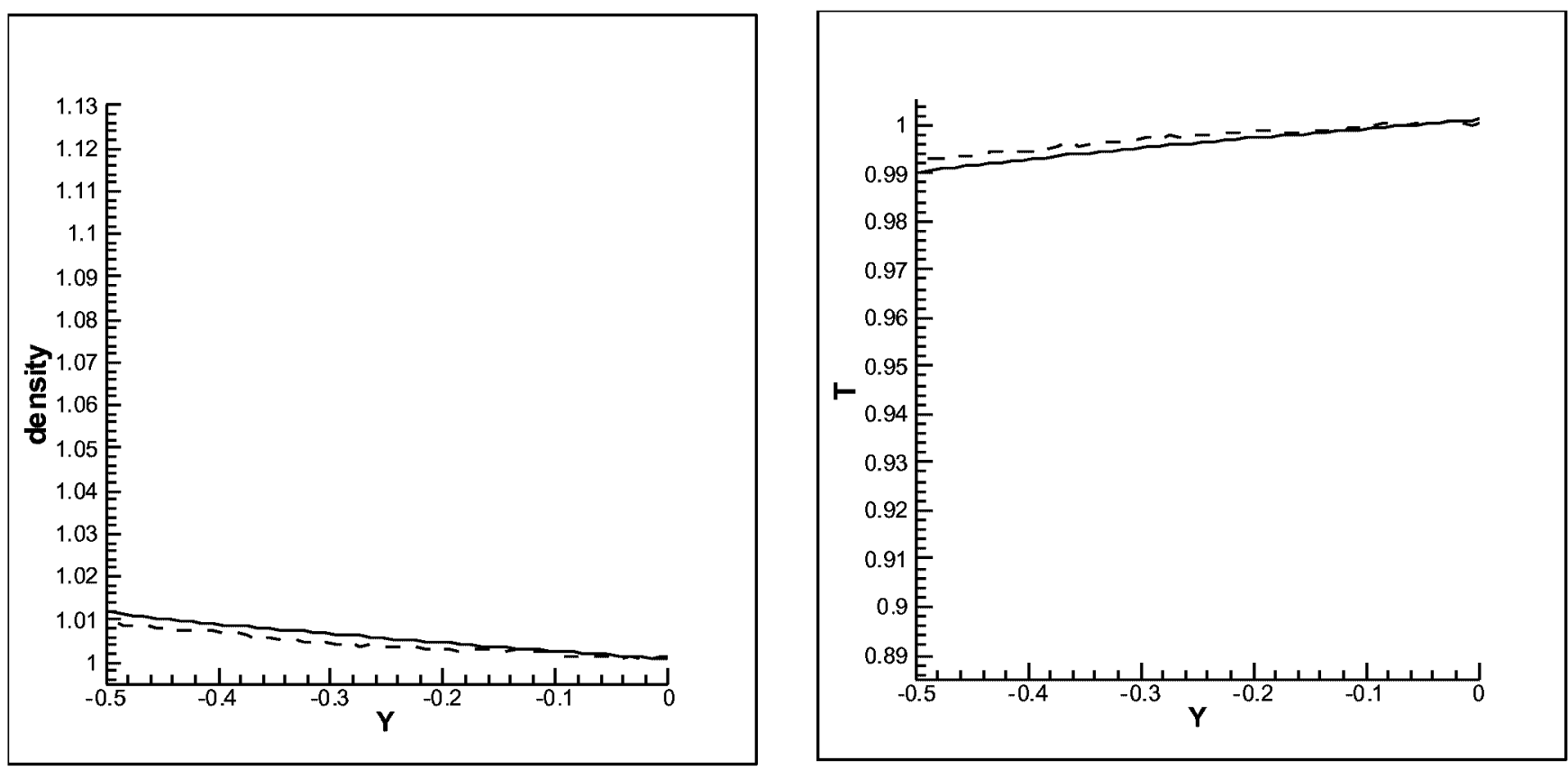

FIG. 3. Density and temperature distribution for $\mathrm{Kn}=0.7582$ with $\mathrm{a} \pm=0.826$ and $\Delta T / T_{0}=0.03$.

Here, - : IP result; -----: DSMC calculation.

\section{ACKNOWLEDGEMENT}

The support by NNSFC (grants 19889209,59876043 ) is cordially appreciated by the authors.

\section{REFERENCES}

1. Bird, G. A., Molecular Gas Dynamics and the Direct Simulation of Gas Flows, Clarendon Press, Oxford (1994).

2. Fan, J., and Shen, C., "Statistical Simulation of Low-Speed Unidirectional Flows in Transition Regime", in Rarefied Gas Dynamics, ed. by R. Brun et al., vol.2, 1999, pp 245-252.

3. Fan, J., Boyd, I. D., and Cai, C. P., "Computation of Rarefied Flows Around a NACA 0012 Airfoil", AIAA Paper 99-3804 (1999).

4. Liu, H. L., Xie, C., Shen, C., and Fan J.,"Microchannel flow with diaphrag", presented at this symposium.

5. Gross E. P. and Ziering S., "Heat Flow between Parallel Plates", in Phys. Fluids 2, 701(1959).

6. Ohwada, T., "Heat Flow and Temperature and Density Distributions in a Rarefied Gas between Parallel Plates with Different Temperatures. FiniteDifference Analysis of the Nonlinear Boltzmann Equation for Hard-Sphere Molecules", in Phys. Fluids 8(8), 2153(1996).

7. Teagan, W.P. and Springer, G.S., "Heat-Transfer and Density-Distribution Measurements between Parallel Plates in the Transition Regime", in Phys. Fluids 11,497(1968). 